\title{
Desarrollo de hormigones con aglomerante cal-puzolana fina como material cementicio suplementario
}

\section{D evelopment of concrete mixtures with fine lime-pozzolan binder as supplementary cementitious material}

\author{
Juan José Dopico*1, Fernando Martirena Hernandez*, Robert L. Day**, Bernhard Middendorf***, Matthias G ehrke***, Lesday
} Martinez*

* Universidad Central Marta Abreu de las Villas (U CLV), CU BA
** University of Calgary, CANADÁ

*** U niversity of Kassel, ALEM ANIA

Resumen

Fecha de recepción: 22/ 05/ 2008 Fecha de aceptación: 30/ 10/2008 PAG. 171 - 178

\begin{abstract}
La alta resistencia y durabilidad alcanzada en los hormigones de alta y ultra alta resistencia está asociada a la obtención de una matriz muy densa, definida a partir del uso de altos volúmenes de adiciones minerales muy finas, tales como las cenizas volantes, microsílice, metacaolín y polvos finos de cuarzo. El trabajo muestra un estudio sobre el uso del aglomerante cal- puzolana (ACP) de alta finura, como adición mineral activa en el hormigón. Las partículas muy finas de la cal, de tamaños entre las 0.1-10 $\mu$ m pueden llenar los vacíos entre los granos de cemento, mientras las partículas más grandes de la puzolana, entre 10-100 $\mu \mathrm{m}$, pueden llenar los vacíos entre los granos más finos del agregado, resultando en una matriz más densa. La adición de hidróxido de calcio $(\mathrm{HC})$ durante el mezclado del hormigón, también incrementa la concentración de iones $\mathrm{Ca}^{+2}$ y $\mathrm{OH}^{*}$, lo $\mathrm{Cual}$ resulta en una mejor y mas rápida hidratación del Cemento Pórtland Ordinario (CPO) y de la reacción puzolánica. El uso de la ACP como adición mineral activa en hormigones de alta resistencia (HAR), podría contribuir a disminuir los costos del producto sin afectar su resistencia mecánica y durabilidad usando menos cemento, con lo cual se estaría mejorando el perfil ecológico de este material. Resultados iniciales son mostrados en este trabajo, requiriéndose de ensayos adicionales para establecer los beneficios del uso de la ACP en los HAR. Un ejemplo de aplicación de este trabajo en hormigones son también presentados.
\end{abstract}

Palabras Clave: Adición mineral, Adición cal- puzolana, resistencia a la compresión, cal, puzolana, hormigón

Abstract

The very high concrete strength and durability achieved in high and ultra-high-performance concrete is associated with a very dense matrix, accomplished through the use of high volumes of very fine mineral additions, such as fly ash, silica-fume, metakaolín and ground quartz sand. The paper reports on a study where a finely ground lime-pozzolan binder (LPB) is used as active mineral addition in concrete. The very fine lime particles, having size between 0.1-10 $\mu \mathrm{m}$, can fill the gaps between cement grains, while the larger pozzolan particles, having size between 10-10qum, can fill the gaps between fine aggregate grains; this results in a much denser matrix. The addition of lime during concrete mixing also increases the $\mathrm{Ca}^{+2}$ and $\mathrm{OH}^{-}$ion concentration, which results in a better and faster hydration of both ordinary portland cement $(O P C)$ and pozzolanic reaction products. The use of $L P B$ as an active addition in some ultra-high performance concretes could lowe the cost of the product for equivalent strength and durability performance, through the use of less cement, thus improving the ecologic profile of the material. Results from an initial series of tests are examined in this paper; further testing is required to establish the benefits of the use of LPC in High Performance Concrete. Examples of applications of this work in concretes are also presented.

Keywords: Mineral addition, lime-pozzolan binder, compression strength, lime, pozzolan, concrete

\section{Introducción}

El cemento Pórtland ha contribuido sustancialmente al desarrollo económico de la sociedad moderna, su fabricación sustentada en el uso de recursos no renovables ha generado la producción de grandes cantidades de gases de efecto invernadero. La producción mundial de cemento Pórtland ha experimentado un

${ }^{1}$ Autor de correspondencia / Corresponding author: Carr. a Camajuaní, km 5,5. Santa Clara. Villa Clara. Cuba Teléfono: 5342 281561: CP 54830, E-mail: dopico@uclv.edu.cu espectacular crecimiento, en el 2000 fue de 1,75 x 109 toneladas, con un por ciento de crecimiento anual del $3.5 \%$ desde 1970 . Estas producciones continuaran incrementándose en los próximos años pudiendo llegar a incrementos entre $120 \%$ a $180 \%$ en el 2020 .

Las medidas a tomar para disminuir las emisiones de $\mathrm{CO}_{2}$ durante la producción de cemento pueden ser clasificadas en dos grandes grupos, el primero con el aumento de la eficiencia del proceso de producción 
disminuyendo los consumo de petróleo y el segundo, reduciendo la producción de clinker, a través de la incorporación de las adiciones minerales durante el proceso productivo ó en el momento de la fabricación del hormigón. (Price et al., 1999; Vanderley 2002) La contribución de cualquiera de estas medidas en la reducción del consumo de energía y las emisiones son pequeñas comparadas con las proyecciones estimadas en la producción de cemento en el futuro. Sin embargo la meta es reemplazar hasta un $50 \%$ de combustible fósil, en términos prácticos solo el $15 \%$ ha sido alcanzado hasta ahora. (Schmidt, 2003) Por otra parte, el uso reportado de las adiciones minerales ha llegado solo a un $22 \%$ de reducción de las emisiones. Las mejorías tecnológicas dependen del nivel económico del país donde la producción tiene lugar. [Schmidt, 2002]

A fin de tener similares niveles de consumo de energía y emisiones en el 2014 que los actuales, la industria cementera esta obligada a reducir las emisiones en un $50 \%$. Esto requerirá un cambio de paradigma en la producción y uso del cemento Pórtland para hacer cumplir los requerimientos medio ambientales vigentes. Una solución del problema incluye la reducción de cantidades de cemento Pórtland como material aglomerante para la confección del hormigón.

Progresos en el conocimiento de la ciencia del hormigón, el uso de modernas técnicas en la investigación de la química y microestructura del hormigón, así como del desarrollo de la industria de los aditivos químicos, han abierto nuevas perspectivas para el uso del hormigón como un material de construcción moderno. Estos resultados han revelado nuevas caminos hacia el desarrollo de hormigones de altas resistencias y durabilidad con pocas cantidades de cemento Pórtland. El uso de pequeñas cantidades de cemento Pórtland y grandes cantidades de adiciones puzolánicas y otros subproductos combinados con aditivos de alto poder dispersante, son vistos como una atractiva vía para mejorar el perfil medio ambiental del hormigón.

En la actualidad existe una amplia variedad de cementos mezclados. Los materiales inorgánicos que son usados para reducir el consumo de cemento pueden ser mezclados y/o molidos íntimamente con el clinker y/o mezclados durante la fabricación de este o mezclados durante la fabricación del hormigón o morteros. Las cenizas volantes, escorias granuladas, micro sílice y otras puzolanas naturales o calcinadas son reportados como las adiciones minerales más utilizadas. La naturaleza de los productos formados durante la reacción puzolánica en los cementos mezclados depende de las propiedades de las puzolanas y del clinker utilizado para confeccionar el hormigón. Los principales productos de reacción son el silicato cálcico hidratado (CSH) y pequeñas cantidades de estringita, hidrogranate y aluminatos hidratados. La relativa proporción de los productos de reacción dependen de las características químicas y mineralogía de las puzolanas usadas. (Day, 1992; Taylor, 1993).

Poco se conoce acerca de las reacciones y modo de reacción en una solución supersaturada de $\mathrm{HC}$ que puede existir cuando se use aglomerante calpuzolana o cemento mezclado, uno u otro. Aparentemente, el exceso, crea una situación de nucleación de $\mathrm{HC}$ adicional no disuelto, principalmente debido a la alta superficie específica de este. La presencia temprana de $\mathrm{HC}$ parece acelerar la reacción puzolánica, probablemente debido a la gran cantidad de ión $\mathrm{Ca}^{2+}$ aprovechable en la solución. (Williams et al., 2002)

El uso del aglomerante Cal- Puzolana como adición mineral activa en el hormigón, podría contribuir a disminuir los costos del producto sin afectar su resistencia mecánica y durabilidad utilizando menos cemento, con lo cual se estaría mejorando el perfil ecológico de este material. Resultados iniciales se muestran en este trabajo, requiriéndose de ensayos adicionales para establecer los beneficios del uso de la ACP en los hormigones. Un ejemplo de aplicación de este trabajo en hormigón es también presentado.

\section{Discusión y desarrollo}

\subsection{U so de adiciones minerales en el hormigón}

Las adiciones minerales de alta finura pueden ayudar al mejoramiento de las cualidades del hormigón. Estos pueden ser efectos físicos tal como el incremento de la compacidad, o efectos físico- químicos como los nuevos productos de reacción formados durante la reacción puzolánica. En ambos casos el efecto final es similar, la porosidad del hormigón disminuye y la distribución y tamaño de los poros se hacen más pequeños. El uso de las puzolanas puede modificar las propiedades reológicas, mecánicas y la durabilidad del hormigón.

En hormigones de resistencias normales, las puzolanas son añadidas para reducir los costos y mejorar los valores de resistencia y durabilidad de la masa endurecida. En tales casos, las puzolanas ayudan a mejorar la compacidad de los sólidos, pero el rol primario es 
proveer silicato cálcico hidratado adicional por medio de la reacción con el agua y con el hidróxido cálcico proveniente de la reacción del cemento Pórtland. Esta reacción puzolánica es pequeña para la mayoría de las puzolanas utilizadas en altas proporciones, así, los beneficios son observados en el plazo de una semana a varias semanas después de mezcladas. Algunas de estas puzolanas altamente reactivas (tales como la silica fume) son añadidas en pequeñas proporciones y ayudan a mejorar los valores de resistencias a edades tempranas así como también la durabilidad a mayores edades. (Shannang y Yeginobali, 1995; Singh, 2000).

En hormigones de alta y ultra- altas prestaciones, el fin primario es optimizar la distribución de tamaño de partículas, especialmente las finas. El uso de plastificantes de alto poder dispersante permiten obtener mezclas con baja relación agua- aglomerante. El hormigón resultante tiene una alta resistencia, alta compacidad y por ende baja porosidad. En muchas de estas mezclas, sin embargo, la sustitución de cemento Pórtland es menos del 15\% . (Malhotra y Mehta, 1996; Zhang et a.l., 1996; Aitcin, 2000). Conservadoramente, en hormigones con altos volúmenes de cenizas volantes, las adiciones minerales son mucho mas altas que en los hormigones de cemento pórtland ordinario, y la relación agua/aglomerante mucho mas baja (en el orden de 0,35 ). La resistencia a los 28 días está en el rango de los 60-90 M Pa, muy lejos de los valores obtenidos en hormigones con el $100 \%$ de CPO. (Bouzoubaa et al., 1998; Lam et al, 2000; Poon et al, 2000).

Las adiciones minerales ó puzolánicas ejercen una doble función en estos casos. Las finas partículas puzolánicas llenan los espacios vacíos entre los granos de cemento y entre el resto de los granos puzolánicos mejorando la compacidad. Solo una pequeña parte de las adiciones puzolánicas, menos del $20 \%$ reacciona. La resistencia a la compresión, sin embargo, no se corresponde con el bajo nivel de hidratación alcanzado, es atribuida a la contribución de la interacción eléctrica entre las partículas más finas de las cenizas volantes. (Lam et al., 2000; Q ualin et al., 2003).

La reacción puzolánica en muchas puzolanas es significativa después de los 7 días, cuando la mayoría de los productos de reacción del cemento ya se han formado y la concentración al calina es considerable para romper los enlaces y facilitar la formación de productos de reacción del cemento. En muchos casos y dependiendo de la reactividad de la puzolana, muchas de las reacciones finalizan en los primeros 60 días. (Shannang y Yeginobali,
1995; Jamal, 1995; Malhotra y M ehta, 1996). Sin embargo, el uso de grandes volúmenes de adiciones puzolánicas incrementan el riesgo de la auto-neutralización debido al excesivo consumo de hidróxido de calcio durante la reacción puzolánica. Una caída significativa en el pH, puede causar la disolución de otros productos de reacción y la destrucción de la matriz cementicia. (Groves y Richardson, 1994).

\subsection{El Aglomerante Cal- Puzolana como adición mineral} activa en el hormigón

Las propiedades del hormigón con grandes volúmenes de sustitución pueden ser mejoradas sustituyendo el cemento por aglomerante cal-puzolana, que con solo puzolanas. La presencia de cal adicional, reduce el riesgo de autoneutralización, aun para grandes volúmenes de sustitución. La presencia de cal incrementa la concentración de iones $\mathrm{Ca}^{2+}$, lo anterior se fundamenta en su contribución temprana en la formación de productos de reacción. La presencia de cal conduce al incremento de iones $\mathrm{OH}^{-}$, cuya tarea es romper los enlaces silicios en la puzolana, y con ello acelerar el comienzo de la reacción puzolánica.

Los efectos de la cal pueden ser vistos en tres etapas diferentes:

1. Durante el mezclado, las finas partículas de cal ocupan los espacios vacíos entre los granos de cemento y limitan el flujo de agua, de este modo incrementan la retención de agua en el hormigón fresco, actuando como un agente dispersante que previene la floculación e incrementa la plasticidad de la mezcla. (Swamy, 1986; Malhotra y Mehta, 1996).

2. A edades tempranas la cal ayuda al incremento de la compacidad del hormigón, dado que los granos de cal por ser tan finos, no han sido completamente disueltos y llenan los huecos entre los granos de cemento.

3. A mayores edades, las finas láminas hexagonales de $2 \mu \mathrm{m}$ a $5 \mu \mathrm{m}$ ocupan los espacios entre los productos de reacción, tal como se muestra en la Figura 1 , foto obtenida en el SEM de una muestra a los 28 días de una pasta donde el $60 \%$ de CPO ha sido sustituido por aglomerante cal-puzolana. Las láminas hexagonales son altamente visibles muy cerca de la fase CSH. 


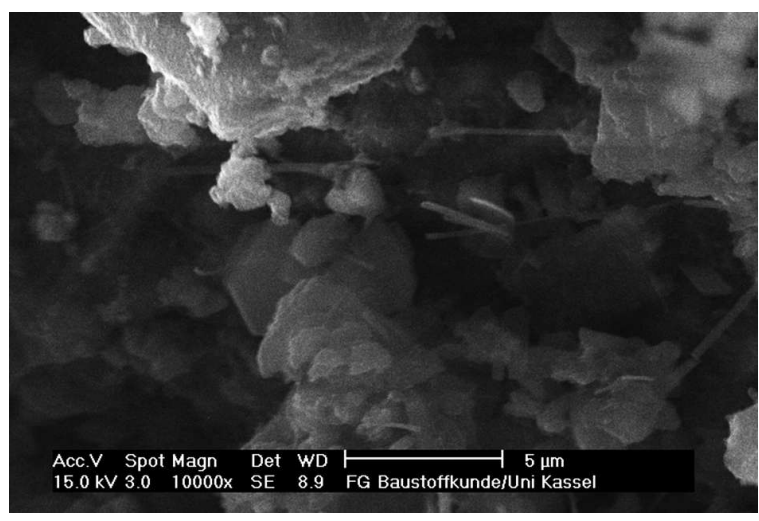

Figura 1. Láminas hexagonales de HC observadas en el SEM, pasta de CPO / ACP, 28 días

El aglomerante Cal- Puzolana usado en el presente trabajo consiste en una mezcla de $20 \%$ de cal y $80 \%$ de puzolana en peso, que mezclados y molidos de forma conjunta por un tiempo de una hora hasta fino polvo, alcanza finura similar a la del CPO. La puzolana utilizada fue una toba zeolítica (Zeolita) obtenida de yacimientos abundantes en Cuba.

A través del proceso de molienda, la distribución por tamaño de partículas de la puzolana es mejorada, compensando el efecto de la forma irregular de los granos. La interrelación entre la cal y la puzolana durante la molienda es también de gran interés. La cal, al ser mucho mas blanda que la puzolana, alcanza mayor finura. (Martirena, 1994). La Figura 2, representa la distribución granulométrica obtenida por granulometría láser de los polvos utilizados, donde puede ser apreciado el tamaño de los granos, la cal entre los 1 - $30 \mu \mathrm{m}$, las puzolana mucho mas grandes, entre las 10-100 $\mu \mathrm{m}$.

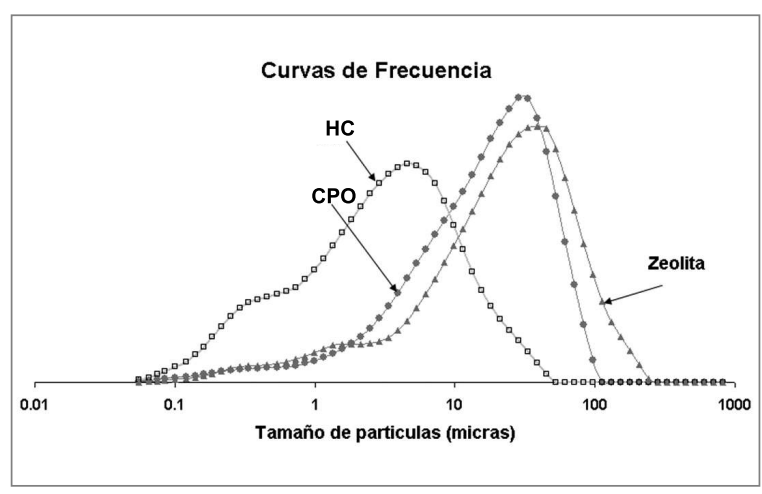

Figura 2. Distribución granulométrica CPO, HC, y zeolita

La Figura 3 muestra los resultados obtenidos en el ensayo de Conductimetría, donde se aprecia perdida de conductividad en el tiempo, lo cual nos permite inferir la reactividad de la puzolana estudiada en la solución de hidróxido de calcio.

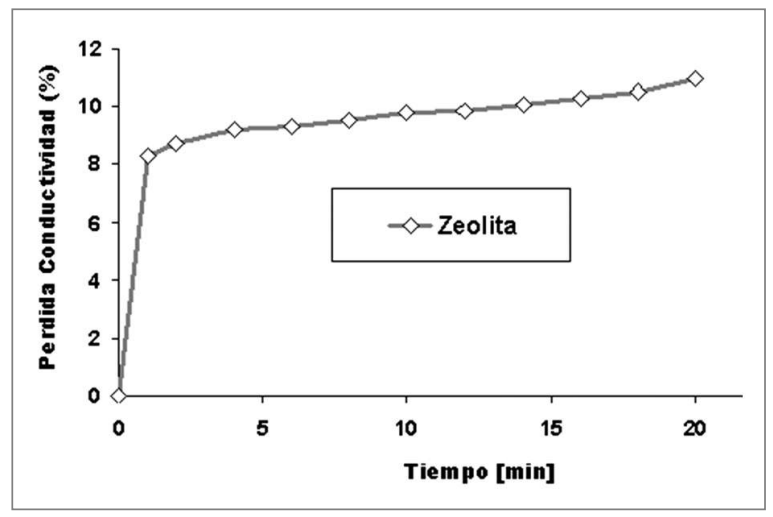

Figura 3. Pérdida de conductividad en la toba zeolítica

2.3 Hormigón con altos volúmenes de adición CalPuzolanas

Con el objetivo de optimizar los consumos de CPO en mezclas de hormigón, la relación que se establece entre la resistencia a la compresión y el contenido de cemento debe ser bien interpretada. U na matriz cementicia más densa y mas fuerte, no será alcanzada con solo incrementar las cantidades de CPO, mayores cantidades de cemento, traería incrementos en la demanda de agua de amasado y con ello de la porosidad y permeabilidad en la matriz de hormigón endurecido. En cambio, usando adiciones minerales de alta finura, como sustitutas parciales de los contenidos de CPO en combinación con superplastificante permitirían trabajar mezclas de hormigón con baja relación agua/ aglomerantes. Esto traería consigo, disminución de micro-fisuras asociadas a la retracción, y garantizaría mejores condiciones para la hidratación del CPO usado. De igual forma, los granos de la adición complementarían la distribución granulométrica de los finos, pudiendo ocupar los espacios vacíos entre los granos de cemento y potencialmente los espacios existentes entre los pequeños granos del agregado. Este principio puede ser aplicado a cualquier tipo de hormigón, tal como ha sido aplicado en la obtención de los hormigones autocompactantes y de alta y ultra altas resistencias, con la ventaja de alcanzar excelentes propiedades mecánicas y durabilidad con reducciones notables en los consumos de CPO.

Para valorar cuanto CPO puede ser sustituido sin variar las propiedades reológicas de las mezclas, los autores proponen un método que permite corregir las proporciones de las mezclas diseñadas tradicionalmente, incorporando altos volúmenes de adición mineral calpuzolana. La propuesta de corrección se compone de 
dos etapas principales.

La primera etapa consiste en la determinación de la cantidad de CPO en peso, que puede ser sustituido por adición mineral cal-puzolana, en combinación con superplastificante, sin provocar cambios significativos en las propiedades reológicas de la mezcla, para ello, mezclas experimentales serán confeccionadas, realizando sustituciones parciales de los contenidos de CPO por igual peso de adición cal- puzolana, por ejemplo 10\%, $20 \%, 30 \%$, ajustando las cantidades de agua para cada mezcla a fin de mantener la consistencia dentro del rango. Ensayos de resistencia de probetas cilíndricas a edades tempranas serán realizados para cada mezcla.

El límite de sustitución de CPO en peso, por adición mineral cal- puzolana lo definirá la mezcla donde los valores de resistencia a la compresión no ofrezcan cambios significativos en relación a los expresados por la mezcla control y donde la relación a/aglom no se incremente significativamente (menos de un 20\%). El volumen de pasta $(\mathrm{Vp})$ que defina esta mezcla se considera como adecuado para garantizar los valores de consistencia y resistencia a la compresión requeridos en una mezcla con sustitución parcial de los contenidos de CPO.

El uso de la ACP provoca cambios en la relación agregado/pasta. La adición mineral al tener menor densidad que el CPO incrementa el volumen de pasta en la mezcla, incrementando también la separación entre los granos del agregado, en la Figura 4, se observa claramente, que en un mortero donde se ha reemplazado un $40 \%$ de CPO, la separación entre los granos del árido ha aumentado debido al incremento del volumen de pasta.

La segunda etapa comprende la determinación de la cantidad de CPO en volumen, que puede ser sustituido por adición mineral cal- puzolana en combinación con superplastificante, sin provocar cambios en los valores de resistencia a la compresión y la consistencia deseados. En esta etapa, manteniendo constante el volumen de pasta determinado en el paso anterior, de manera que la relación volumétrica agua/finos se mantiene constante tal como lo proponen Bornemann y Schmidt (Bornemann, 2002; Schmidt, 2003), y el resto de los constituyentes también constantes, se varían las proporciones en volumen de CPO por ACP, para determinar la mezcla en la cual la resistencia a la compresión y la consistencia definan los valores esperados. Esta mezcla permitirá establecer la cantidad mínima de cemento necesaria.

Es de suponer, que mientras más reactiva sea la puzolana que se utilice, mayor será la posibilidad de reducir los consumos de CPO dentro de este volumen de pasta constante, dado por el aporte de la adición en productos hidratados.

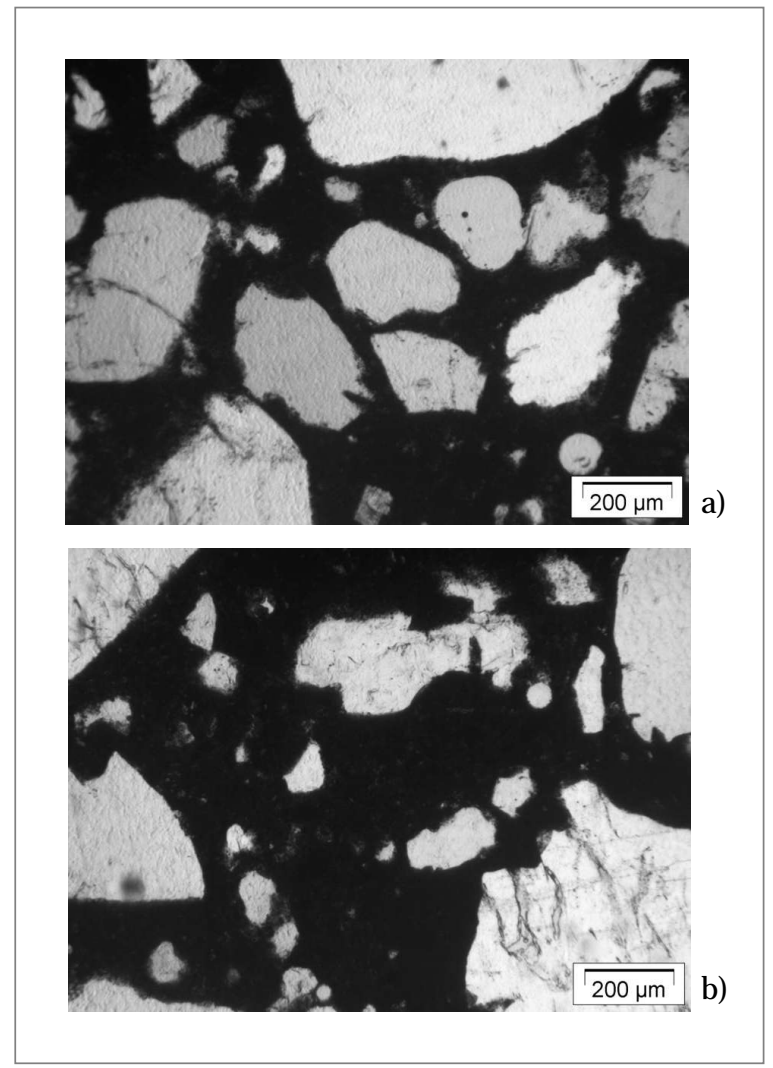

Figura 4. Morteros $100 \%$ CPO (a) y $40 \%$ ACP (b)

2.4 Ejemplo de aplicación de la propuesta de sustitución de CPO por ACP

Como ejemplo de aplicación de los principios descritos en el presente trabajo, fue trabajada una mezcla de hormigón estructural diseñada para la obtención de $30 \mathrm{MPa}$ de resistencia a la compresión a los 28 días con $12 \pm 1 \mathrm{~cm}$ de asentamiento medidos en el Cono de Abrams. Áridos triturados de procedencia caliza, tamaño máximo $9.52 \mathrm{~mm}$ para el árido grueso, 2.56 modulo de finura para el árido fino, cemento portland P-350 según la normativa cubana NC 95, aditivo químico Mapefluid N-200, superplastificante tipo F según ASTM C- 494. Adición mineral conformada por un $20 \%$ en peso de hidróxido de calcio (72\% reactiva), y $80 \%$ en peso de toba zeolítica, molturadas de forma conjunta en molino de bolas durante una hora, alcanzando una finura del $12 \%$ en el tamiz 170. La Tabla 1 muestra el análisis químico y físico de los aglomerantes utilizados. 
Tabla 1. Análisis químico y físico aglomerantes

\begin{tabular}{||cccc||}
\hline & $\mathrm{CPO}$ & Cal & Zeolita \\
\hline Químicos (en masa) & & & \\
$\mathrm{SiO}_{2}$ & 70.4 & 0.88 & 74.68 \\
$\mathrm{Fe}_{2} \mathrm{O}_{3}$ & 1.38 & 0.29 & 2.87 \\
$\mathrm{Al}_{2} \mathrm{O}_{3}$ & 2.42 & 0.34 & 12.69 \\
$\mathrm{CaO}$ & 9.76 & 97.42 & 4.89 \\
$\mathrm{MgO}$ & 2.28 & 0.59 & 0.53 \\
$\mathrm{~K}_{2} \mathrm{O}$ & 3.6 & & 1.28 \\
$\mathrm{Na}_{2} \mathrm{O}$ & 0.23 & & 2.9 \\
$\mathrm{SO}_{3}$ & 0.35 & 0.47 & 0.03 \\
Fisicos & & & \\
Sup.Esp. Blaine $\left(\mathrm{cm}^{2} / \mathrm{g}\right)$ & 2974 & 7656 & 3425 \\
Peso Especifico & 3.15 & 2.46 & 2.29 \\
\hline
\end{tabular}

Etapa I: La Tabla 2 recoge los componentes de las mezclas trabajadas desde el $0 \%$ al $40 \%$ de sustitución en masa de CPO por adición mineral cal- zeolita, la Figura 5 muestra los resultados de los ensayos de resistencia a la compresión. La mezcla donde se ha reemplazado el $10 \%$ de los contenidos de CPO produce un incremento en los valores de resistencias a la compresión por encima de los expresados en la mezcla con el $100 \%$ de CPO. En cambio por encima del $30 \%$ existe una caída brusca de la resistencia. La mezcla preparada con el $30 \%$ de sustitución de CPO por adición mineral cal-zeolita no ofrece cambios significativos en los valores de resistencia a los 28 días a los expresados por la serie control, definiéndose entonces como la mezcla que define el límite de sustitución en peso de CPO por adición mineral.

Tabla 2. Proporciones de las mezclas elaboradas en la Etapa I

\begin{tabular}{||ccccccc||}
\hline \multirow{2}{*}{ Materiales } & \multirow{2}{*}{$\mathrm{UM}$} & \multicolumn{5}{c||}{ Proporción ACPICPO (masa) } \\
\cline { 3 - 7 } & $\mathrm{kg} / \mathrm{m} 3$ & 402.12 & 365.58 & 317.65 & 275.66 & 232.84 \\
\hline Cemento & $\mathrm{kg}$ & 0.00 & 40.62 & 79.41 & 118.14 & 155.22 \\
Cal-Puzolana & $\mathrm{kg} / \mathrm{m} 3$ & 159.84 & 145.22 & 157.83 & 158.50 & 164.44 \\
Agua & $\mathrm{kg} / \mathrm{m} 3$ & 159.84 & $\mathrm{~kg} 3$ & \\
Arido Fino & $\mathrm{kg} / \mathrm{m} 3$ & 834.40 & 842.86 & 823.90 & 817.12 & 805.23 \\
Arido Grueso & $\mathrm{kg} / \mathrm{m} 3$ & 940.96 & 950.51 & 929.12 & 921.48 & 908.07 \\
Superplastificante & $\mathrm{kg} / \mathrm{m} 3$ & 4.82 & 4.87 & 4.78 & 4.72 & 4.66 \\
Volumen de Pasta & $\mathrm{l} / \mathrm{m}^{3}$ & 287.50 & 280.47 & 296.19 & 301.84 & 311.70 \\
Relacion a/c (masa) & - & 0.40 & 0.40 & 0.50 & 0.57 & 0.71 \\
Relación a/aglom. & - & 0.40 & 0.36 & 0.40 & 0.40 & 0.42 \\
Asentamiento & $\mathrm{cm}$ & 13 & 12 & 11 & 12 & 12 \\
\hline \hline
\end{tabular}

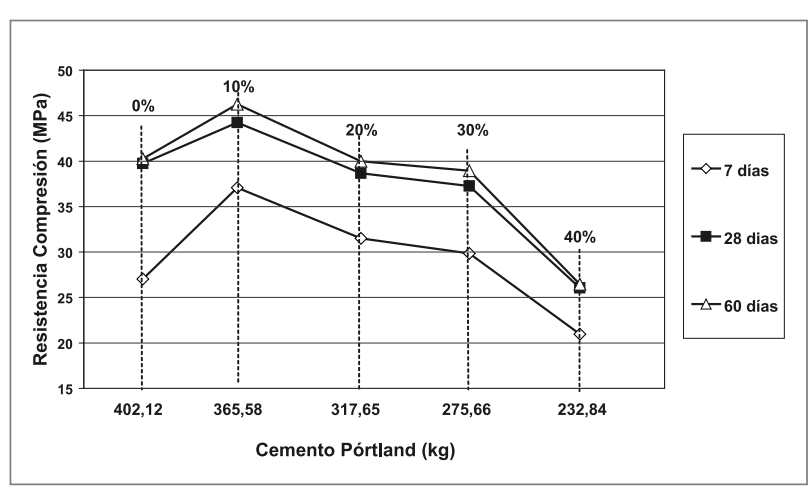

Figura 5. Resultados Resistencia a la Compresión Etapa I
Etapa II: La mezcla del $30 \%$ de sustitución en peso seleccionada en el paso anterior, representa a su vez el $39 \%$ de sustitución en volumen, en lo adelante $40 \%$. Manteniendo constante el volumen de pasta de 301.84 litros, de manera que la relación volumétrica agua - finos sea también constante (1.07), fueron variadas en volumen las proporciones entre CPO y ACP para puntos por encima y por debajo del $40 \%$. La Tabla 3 muestra la dosificación de las mezclas fabricadas y la Figura 6, los resultados de resistencia a la compresión obtenidos

Tabla 3. Proporciones de las mezclas elaboradas en la Etapa II

\begin{tabular}{||ccccccc||}
\hline \multirow{2}{*}{ Materiales } & \multirow{2}{*}{$\mathrm{UM}$} & \multicolumn{5}{c||}{ Proporción ACP/CPO (volumen) } \\
\cline { 3 - 8 } & & $20 \%$ & $30 \%$ & $40 \%$ & $50 \%$ & $60 \%$ \\
\hline Cemento & $\mathrm{kg} / \mathrm{m}^{3}$ & 361.22 & 316.06 & 275.66 & 225.76 & 180.61 \\
Cal-Puzolana & $\mathrm{kg} / \mathrm{m}^{3}$ & 60.93 & 91.42 & 118.14 & 152.37 & 182.84 \\
Agua & $\mathrm{kg} / \mathrm{m}^{3}$ & 158.50 & 158.50 & 158.50 & 158.50 & 158.50 \\
Arido Fino & $\mathrm{kg} / \mathrm{m}^{3}$ & 817.12 & 817.12 & 817.12 & 817.12 & 817.12 \\
Arido Grueso & $\mathrm{kg} / \mathrm{m}^{3}$ & 921.48 & 921.48 & 921.48 & 921.48 & 921.48 \\
Superplastificante & $\mathrm{kg} / \mathrm{m}^{3}$ & 4.72 & 4.72 & 4.72 & 4.72 & 4.72 \\
Volumen de Pasta & $\mathrm{I}$ & 301.84 & 301.84 & 301.84 & 301.84 & 301.84 \\
Relación agua/finos. & - & 1.07 & 1.07 & 1.07 & 1.07 & 1.07 \\
Relacion a/c (masa) & - & 0.44 & 0.50 & 0.57 & 0.70 & 0.88 \\
Relación a/aglom. & - & 0.38 & 0.39 & 0.40 & 0.42 & 0.44 \\
Asentamiento & $\mathrm{cm}$ & 12.00 & 13.00 & 12.00 & 12.00 & 11.00 \\
& & & & & & \\
\hline
\end{tabular}

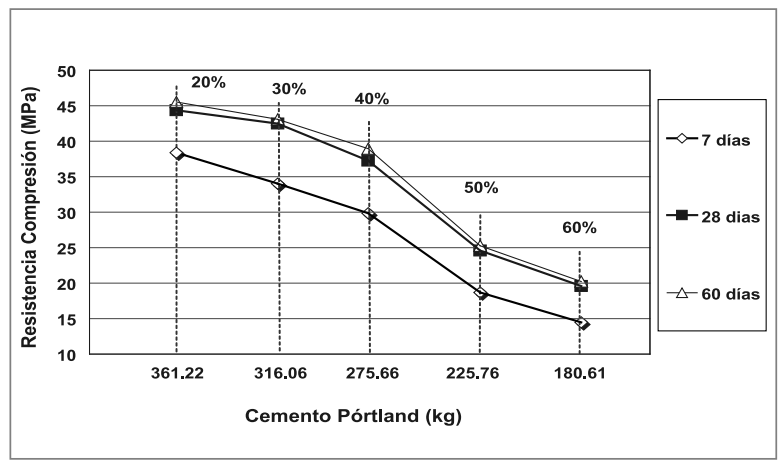

Figura 6. Resultados Resistencia a la Compresión Etapa II

Los resultados obtenidos definen a la mezcla del 40\%, como la mezcla del límite de sustitución en volumen de CPO por adición cal-zeolita, mayores valores de sustitución hacen caer bruscamente los valores de resistencia, definiendo hormigones de resistencias normal pero con notables reducciones en los consumos de cemento, en cambio, menores reemplazos de los contenidos de CPO, definen hormigones de alto desempeño según la normativa cubana NC- 120, para contenidos de CPO aun por debajo de los requeridos por la mezcla control. Todas las mezclas mantienen los valores de consistencia. Los resultados de los ensayos realizados confirman que grandes cantidades de CPO, pueden ser reemplazados por $A C P$, sin afectar los requerimientos de resistencia a los 28 días y la consistencia de un hormigón. 
Los especímenes ensayados en la etapa II, muestran mejor comportamiento en los valores de resistencia a la compresión entre el $20 \%$ y el $30 \%$ cuando se comparan con los resultados de la etapa I para similares contenidos en masa de CPO por metro cúbico. No son apreciables los incrementos de resistencia a la compresión a los 60 días cuando se comparan con los resultados a los 28 días.

\subsection{Influencia de la adición Cal- Puzolana}

Podría pensarse que la adición de cal adicional a la que produce el CPO en su hidratación podría ser perjudicial para la mezcla, pues la presencia de cal libre es un punto de debilidad para matrices en ambientes agresivos. Por esta razón se decidió evaluar la cantidad de cal libre en pastas donde se sustituye parcialmente CPO por ACP.

La Figura 7 muestra los difractogramas de rayos $X$, de pastas fabricadas con diferentes relaciones de CPO/ACP. Puede ser observado que para mayores valores de sustitución de CPO por adición cal-puzolana, menor es la intensidad de los picos de hidróxido de calcio, lo cual sugiere una considerable reactividad puzolánica durante los primeros 28 días de reacción.

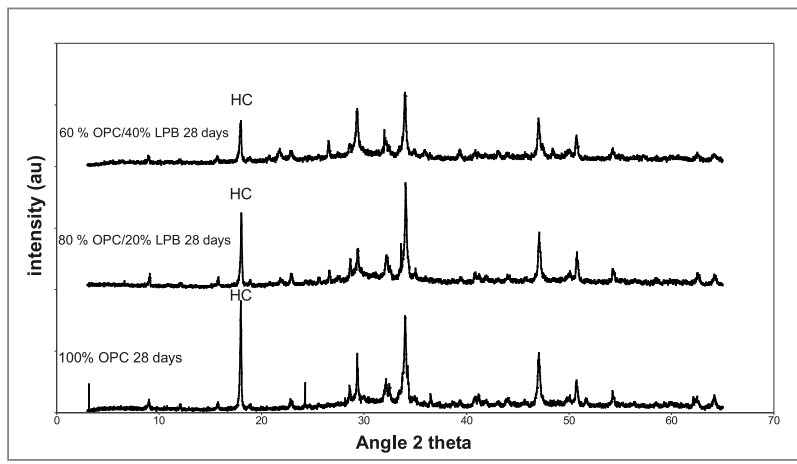

Figura 7. Difractogramas de Rayos $X$ de pastas de CPO hidratado y CPO/ACP

Se decidió verificar este resultado con el análisis de TG de pastas con $0 \%, 20 \%$ y $40 \%$ de sustitución de CPO por ACP, curadas durante 28 días sin exposición al ambiente para evitar carbonatación. Las curvas de perdida de masa contra temperatura, permiten determinar la perdida de agua durante el proceso de deshidroxilación en el rango de los $470{ }^{\circ} \mathrm{C}$ a los $550 \stackrel{\circ}{\circ}$ de temperatura. Conocida las proporciones de las pastas, pueden ser calculados los contenidos teóricos de Hidróxido de calcio en las pastas y con ello el grado de actividad puzolánica. La Tabla 4 refleja los cálculos realizados.
Tabla 4. Determinación consumo Hidróxido de Calcio

\begin{tabular}{|cccccc|}
\hline $\begin{array}{c}\text { CPO } \\
(\mathrm{g})\end{array}$ & $\begin{array}{c}\mathrm{ACP} \\
(\mathrm{g})\end{array}$ & $\begin{array}{c}\text { Masa de } \mathrm{HC} \\
\text { Teórico presente }\end{array}$ & $\begin{array}{c}\text { Masa calculada } \mathrm{HC} \\
\text { presente }\end{array}$ & $\begin{array}{c}\mathrm{HC} \\
\text { consumido }\end{array}$ & $\begin{array}{c}\mathrm{HC} \text { consumido } \\
\text { por }(\mathrm{g}) \mathrm{de} \\
\text { puzolana }\end{array}$ \\
\hline 100 & 0 & 8.34 & 8.34 & 0 & 0 \\
80 & 20 & 10.67 & 8.75 & 1.92 & 0.12 \\
60 & 40 & 13 & 5.34 & 7.66 & 0.24 \\
\hline
\end{tabular}

El análisis de los resultados en la Tabla 4 confirman la presencia de actividad puzolánica, indicando también que esta actividad es mucho más alta en la medida que mas alta es la proporción de ACP usada, la pasta con proporción 60 CPO/ 40 ACP consume $0.24 \mathrm{~g}$ de $\mathrm{HC}$ por gramo de puzolana, mientras que en la relación 80: 20 sólo es consumido $0.12 \mathrm{~g}$. Esto indica que la adición de cal adicionalmente tiene resultados beneficiosos para la matriz, pues aparentemente aumenta el volumen de productos estables resultantes de la reacción puzolánica.

\section{Conclusiones}

El uso de la adición cal- puzolana permite reducir las cantidades de cemento portland usado en los hormigones de alto performance, constituyendo una atractiva propuesta desde el punto de vista medio ambiental. Los resultados de los ensayos reportados en el presente trabajo se corresponden con hormigones de resistencia normal, pero los principios son aplicables también a los hormigones de alta resistencia. Los resultados sugieren que la cantidad de cemento puede ser reducida, sin afectar los valores de resistencia y la consistencia esperados usando el procedimiento descrito, que permite determinar el límite de sustitución de CPO por ACP en combinación con superplastificante trabajando constante la relación volumétrica agua finos dentro de la mezcla.

Desde el punto de vista de la durabilidad, el uso de altos niveles de hidróxido de calcio dentro de la mezcla fresca resulta interesante. Ensayos preliminares indican que la cal añadida se combina con la puzolana para producir nuevos productos estables de reacción, y que la cantidad de cal libre en la matriz no aumenta. Implementación a mayores escalas tecnológicas, sugieren la realización de ensayos adicionales que valoren el comportamiento del transporte de sustancias agresivas dentro de los especimenes bajo diversas situaciones de exposición, a fin de establecer los limites de sustitución de CPO por ACP adecuados a los requerimientos de durabilidad. 


\section{Referencias}

Aitcin P. C. (2000), "Cements of yesterday and today. Concrete of tomorrow", Cement \& Concrete Research Vol. 30, No. 9, pp 1349-1359.

ASTM C 494/C 494M - 99 (1999), Standard Specification for Chemical Admixtures for Concrete, July. Bouzoubaa N., Zhang M. H., Bilodeau A. y Malhotra V. M. (1998), "Laboratory-produced high-Volume fly ash blended cements: physical properties and compressive strength of mortars", Cement \& Concrete Research, Vol. 28, No. 11, pp 1555-1569.

Bornemann R. (2002), The role of pow ders in concrete. 6 th international Symposium on utilization of high strength / high performance concrete. Leipzig. Day L. (1992), Pozzolans for use in low cost housing: state of the art report. Department of Civil Engineering. Universidad de Calgary. Report N 0. CE92-1. January. Erdogdu K. (1998), "Effect of fly ash particle size on strength of Portland cement fly ash mortars", Cement $\&$ Concrete Research Issue. Vol. 28, No. 9, pp 11091117.

Groves G.W y Richardson I.G. (1994), Microcrystalline Calcium Hydroxide in Pozzolanic Cement Pastes, Cement and Concrete Research, Vol. 24, No. 6, pp 1191-1196.

Jamal Shannag M. (1995), "Properties of pastes, mortars and concrete containing natural pozzolana", Cement $\&$ Concrete Research. Vol. 25, No. 3, pp 647-657.

Lam L., Wong L. y Poon C. S. (2000), “D egree of hydration and gel/space ratio of high Volume fly ash/cement systems", Cement \& Concrete Research Vol. 30, No. 5, pp 747-756.

Malhotra V.M y Mehta P.K. (1996), Pozzolanic and cementitious materials. Published by Gordon and Breach. UK. 1996.

Martirena J.F. (1994), The Development of Pozzolanic Cement in Cuba, Journal of Appropriate Technology, Vol. 21, No. 2, September, Intermediate Technology Publications, U.K.

NC 95 (2001), Cemento Portland. Especificaciones, Vig, Marzo.

NC 120 (2007), Hormigón Hidráulico. Especificaciones. Vig, Noviembre.

Price L., Worrel E. y Phylipsen D. (1999), Energy U se and Carbon Dioxide Emissions in Energy-Intensive Industries in Key developing Countries. Proceedings of the 1990 Earth Technologies Forum, Washington, DC, September 27-29.

Poon C.S., Lam L. y Wong L. (2000), “A study on high strength concrete prepared with large Volumes of low calcium ash", Cement \& Concrete Research Vol. 30 No. 3, pp 447-455.

Q ualin Niu, N aiqian Feng, Jing Yang, Xiaoyan Zheng (2003), Effect of superfine slag powder on cement properties. Cement $\&$ Concrete Research Vol. 32, No. 4, pp 615-621.

Schmidt M. (2002), Secondary Fuels and Raw Materials for Cement. Benefit for the environment and cost reduction. Keynote presentation at IV Simposium on Structures and Materials, november. Santa Clara. Cuba.

Swamy R.N (1986), Cement replacement materials. Series "Concrete technology and Design", Volume 3. Surrey University Press. UK.

Shannang M.J y Yeginobali A. (1995), Properties of pastes, mortars and concretes containing natural pozzolans. Cement \& Concrete Research Vol. 25, N o. 3, pp 647657.

Singh N.B (2000), "Hydration of bagasse-ash blended Portland cement", Cement \& Concrete Research Vol. 30, No. 9, pp 1485-1488.

Schmidt, M. (2003), Ultra-HochleistungsbetonAusganstoffe, Eigenschaften und Leistungsfaehigkeit. Proceedings of the conference UItra-Hochfester Beton University of Kassel, September. Germany. Taylor H.F.W. (1993), Cement Chemistry. Academic Press. London.

Taylor M. R., Lydon F. D. y Barr B. I. (1996) “Mix proportions for high strength concrete", Construction and Building Materials Vol. 10, No. 6, pp 445-450.

Williams P., Biernacki J., Walker R., M eyer M., Rawn J.

y Jianming B. (2002), M icroanalysis of alkali-activated fly ash- $\mathrm{CH}$ pastes. Cement $\&$ Concrete Research Vol. 32, No. 6, pp 963-972. USA.

Vanderley M. (2002), O $n$ the sustainability of the Concrete. Extended version of the paper commissioned by UNEP Journal Industry and Environment.

Zhang M. H., Lastra R. y Malhotra V. M. (1996), "Ricehusk ash paste and concrete: some aspects of hydration and the microstructure of the interfacial zone between the aggregate and paste", Cement \& Concrete Research Vol. 26, No. 6, pp 963-977. 